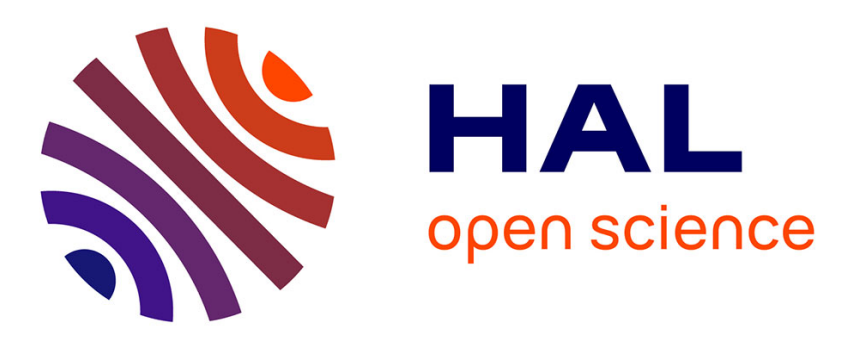

\title{
Cyclostationarity-based versus eigenvalues-based algorithms for spectrum sensing in cognitive radio systems: Experimental evaluation using GNU radio and USRP
}

Amor Nafkha, Babar Aziz, Malek Naoues, Adrian Kliks

\section{To cite this version:}

Amor Nafkha, Babar Aziz, Malek Naoues, Adrian Kliks. Cyclostationarity-based versus eigenvaluesbased algorithms for spectrum sensing in cognitive radio systems: Experimental evaluation using GNU radio and USRP. 2015 Eight International Workshop on Selected Topics in Mobile and Wireless Computing, Oct 2015, Abu Dhabi, United Arab Emirates. 10.1109/WiMOB.2015.7347977 . hal01262070

\section{HAL Id: hal-01262070 \\ https://hal.science/hal-01262070}

Submitted on 4 Feb 2016

HAL is a multi-disciplinary open access archive for the deposit and dissemination of scientific research documents, whether they are published or not. The documents may come from teaching and research institutions in France or abroad, or from public or private research centers.
L'archive ouverte pluridisciplinaire HAL, est destinée au dépôt et à la diffusion de documents scientifiques de niveau recherche, publiés ou non, émanant des établissements d'enseignement et de recherche français ou étrangers, des laboratoires publics ou privés. 


\title{
Cyclostationarity-Based Versus Eigenvalues-Based Algorithms for Spectrum Sensing in Cognitive Radio Systems: Experimental Evaluation Using GNU Radio and USRP
}

\author{
Amor Nafkha*, Babar Aziz ${ }^{\dagger}$, Malek Naoues* ${ }^{*}$ and Adrian Kliks $\ddagger$ \\ ${ }^{*}$ CentraleSupélec/IETR, Avenue de la Boulaie, 35576 Cesson Sévigné, France, email: \{amor.nafkha malek.naoues\}@ centralesupelec.fr \\ $\dagger$ IFSTTAR,LEOST, F-59650 Villeneuve d'Ascq, France, email: babaraziz11@gmail.com \\ $\ddagger$ Poznan University of Technology, Polanka 3, 60-965 Poznan, Poland, email: akliks@et.put.poznan.pl
}

\begin{abstract}
Spectrum sensing is a fundamental problem in cognitive radio systems. Its main objective is to reliably detect signals from licensed primary users to avoid harmful interference. As a first step toward building a large-scale cognitive radio network testbed, we propose to investigate experimentally the performance of three blind spectrum sensing algorithms. Using random matrix theory to the covariance matrix of signals received at the secondary users, the first two sensing algorithms base their decision statistics on the maximum to minimum eigenvalue ratio and the sum of the eigenvalues to minimum eigenvalue ratio, respectively. However, the third algorithm is based on cyclostationary feature detection and it uses the symmetry property of cyclic autocorrelation function as a decision policy. These spectrum sensing algorithms are blind in the sense that no knowledge of the received signals is available. Moreover, they are robust against noise uncertainty. In this paper, we implement spectrum sensing in real environment and the performance of these three algorithms is conducted using the GNU-Radio framework and the universal software radio peripheral (USRP) platforms. The results of the evaluation reveal that cyclostationary feature detector is effective in finite sample-size settings, and the gain in terms of the SNR with respect to eigenvalues-based detectors to achieve $P_{f a}$ (probability of false alarm) $=0.08$ is at least $4 \mathbf{d B}$.

Index Terms-Cognitive radio, spectrum sensing, random matrix theory, compressive sensing, GNU-Radio, USRP platform.
\end{abstract}

\section{INTRODUCTION}

Spectrum sensing to detect the presence of primary user transmissions is a crucial task for a cognitive radio system, which opportunistically accesses the spectrum once an empty subband is detected. Two basic approaches to spectrum sharing have been considered [1]: spectrum overlay and spectrum underlay. According to the spectrum overlay approach, the secondary users sense and identify unused frequency bands and use them for communication purposes. Thus, the secondary users (SU) are responsible for detecting the unused bands and they should vacate the spectrum as soon as the primary user begins its activities [2]. The underlay approach imposes constraints on the secondary users' transmission power level

This research is supported by the European Commission in the framework of the FP7 Network of Excellence in Wireless COMmunications NEWCOM\# (FP7 Contract Number: 318306) so that it can operates below the noise floor of primary users. In this paper we focus on the experimental evaluation of the overlay spectrum sharing.

The detection of spectral occupancy can be viewed as a binary hypothesis testing problem. The null hypothesis $\mathcal{H}_{0}$ corresponds to the case where only additive noise is present, whereas the alternative hypothesis $\mathcal{H}_{1}$ refers to the case when the PU signal is present along with noise. Based on the latter hypothesis testing model, several spectrum sensing techniques have been proposed so far in literature [3], [4], [5]. These techniques are mainly categorized in two family three categories:

- non-blind techniques which require both primary user signal and noise variance information,

- semi-blind techniques which need just a few parameters, such as the additive noise variance and/or the fundamental cyclic frequency,

- blind techniques which exploits only the received signals without any a priori informations about noise or primary user signal,

In this paper, we will conduct an experimental evaluation by implementing three of the most known blind algorithms in the literature and compare their performance in real environments. The choice of the blind algorithms is motivated by the fact that the main aspect of of a typical cognitive radio is related to autonomously exploiting locally unused spectrum. There have been several blind spectrum sensing techniques proposed in literature. They include wavelet-based detection [4], eigenvaluebased detection [6], second order statistical based detection [7], and symmetry property of cyclic autocorrelation function based detection [8].The purpose of the conducted experiment is to sense the spectrum in a given frequency range and to make a reliable decision on the potential presence of primary user signal within frequency subband. In order to achieve this goal, three blind techniques for spectrum sensing have been implemented using USRP platforms and GNU Radio framework. We focus on sensing algorithms based on eigenvalues of received covariance matrix [6] and cyclostationary feature- 
based detectors [8].

The rest of the paper is organized as follows: in section II, the system model is presented. In section III and section IV, we present the eigenvalue based detection and the cyclostationary feature-based detection methods, respectively. In section $\mathrm{V}$, we describe our testbed implemented to carry out the experiments. Section VI presents the experimental results of the considered algorithms. Finally, conclusions are presented in section VII.

\section{System Model}

We consider the problem of detecting the presence of a primary user signal at a specific frequency band based on the signal observed by the secondary user. Detecting the presence of a primary signal can be treated as a binary hypothesis testing problem [9]. Assuming a frequency-flat fading channel between the primary and secondary users, the sampled signal $r(t)$ received by the secondary users, defined as $r[n]=r\left(n T_{s}\right)$ with $1 / T_{s}$ being the sampling rate, is expressed as

$$
r[n]= \begin{cases}w[n] & \Rightarrow \mathcal{H}_{0} \\ (h * s)[n]+w[n] & \Rightarrow \mathcal{H}_{1}\end{cases}
$$

where $*$ is the convolution operator. $s[n]$ and $h[n]$ stand is the digitally modulated signal of the primary user drawn from a certain modulation and the channel between the primary and secondary users, respectively, $w[n]$ is an additive white Gaussian noise with zero mean and variance $\sigma^{2}$. The objective of the spectrum sensing operation is to decide between null hypothesis $\mathcal{H}_{0}$ and alternative hypothesis $\mathcal{H}_{1}$ based on the observation of the received signal $r[n]$. The detection performance is characterized by two probabilities: probability of detection, $P_{\mathrm{d}}$, where the decision is $\mathcal{H}_{1}$, while $\mathcal{H}_{1}$ is true; and probability of false alarm, $P_{\mathrm{fa}}$, which corresponds to the case where the decision is $\mathcal{H}_{1}$ while $\mathcal{H}_{0}$ is true.

In this paper, three algorithms have been tested and compared through experimental analysis, namely eigenvalue based maximum-minimum eigenvalue detection method [6], energy with minimum eigenvalue detection [10], and a method based on cyclostationarity detection called Symmetry Property of Cyclic Autocorrelation Function [8].

\section{EIGENVALUE BASED DETECTION ALGORITHMS}

In this section we study two spectrum sensing algorithms based on the distribution of eigenvalues in large dimensional random matrix theory [11]. The discrete-time domain received signal $r[m]$ under $\mathcal{H}_{1}$ can be given as

$$
r[m]=\sum_{k=0}^{N_{h}} h[k] s[m-k]+w[m]
$$

where $N_{h}$ is the channel filter length. At the cognitive radio's receiver, the received samples are split into $M$ vectors each of length $N_{s}$. Let us consider the following $M \times N_{s}$ matrix consisting of the stacking of the $M$ vectors.

$$
\mathbf{Y}=\left[\begin{array}{cccc}
r_{1}[1] & r_{1}[2] & \cdots & r_{1}\left[N_{s}\right] \\
r_{2}[1] & r_{2}[2] & \cdots & r_{2}\left[N_{s}\right] \\
\vdots & \vdots & \ddots & \vdots \\
r_{M}[1] & r_{M}[2] & \cdots & r_{M}\left[N_{s}\right]
\end{array}\right]
$$

In the absence of primary user signal $\left(\mathcal{H}_{0}\right.$ : alternative hypothesis) all the received samples are uncorrelated whatever fading channel model. Moreover, the non-diagonal element of the received covariance matrix is theoretically zero, whereas the diagonal elements contain the noise variance. Hence, for a fixed $M$ and $N_{s} \rightarrow \infty$, the sample covariance matrix $\frac{1}{N_{s}} \mathbf{Y Y}^{*}$ converges to the true covariance matrix $\sigma^{2} \mathbf{I}_{\mathbf{M}}$.

In this paper, we assume that the noise is additive white Gaussian noise and, furthermore, the noise and the transmitted signal are uncorrelated. Then if the number of received samples $N_{s}$ are large enough, it can be shown that

$$
\mathbf{R}_{\mathbf{r}}\left(\mathbf{N}_{\mathbf{s}}\right) \approx \mathbf{R}_{\mathbf{r}}=\mathbf{E}\left[\frac{\mathbf{1}}{\mathbf{N}_{\mathbf{s}}} \mathbf{Y} \mathbf{Y}^{*}\right]=\mathbf{H R}_{\mathbf{s}} \mathbf{H}^{*}+\sigma^{2} \mathbf{I}_{\mathbf{M}}
$$

where $\mathbf{R}_{\mathbf{r}}$ and $\mathbf{R}_{\mathbf{s}}$ matrices represent the covariance matrices of the received and transmitted signals, respectively. $\mathbf{I}_{\mathbf{M}}$ is the identity matrix of size.

Let $\lambda_{\max }$ and $\lambda_{\min }$ represent the maximum and minimum eigenvalues of $\mathbf{R}_{r}$, respectively. Now suppose that $\rho_{\max }$ and $\rho_{\min }$ are the maximum and minimum eigenvalues of the matrix $\mathbf{H R}_{\mathbf{S}} \mathbf{H}^{*}$ then

$$
\begin{aligned}
& \lambda_{\max }=\rho_{\max }+\sigma^{2} \\
& \lambda_{\min }=\rho_{\min }+\sigma^{2}
\end{aligned}
$$

If $\mathbf{Z}=\mathbf{H R}_{\mathbf{s}} \mathbf{H}^{*}=\delta \mathbf{I}_{\mathbf{M}}$ then $\rho_{\min }=\rho_{\max }$ where $\delta$ is a positive integer. In practice it is highly unlikely that the matrix $\mathbf{Z}$ will be equal to $\delta \mathbf{I}_{M}$ as mentioned in [6]. Hence, if there is no signal present then $\lambda_{\max } / \lambda_{\min }=1$ otherwise $\lambda_{\max } / \lambda_{\min }>1$. Therefore, this ratio can be used to detect the presence or absence of the signal. Based on the eigenvalues of the received covariance matrix, the following two methods are proposed in literature.

\section{A. Maximum-Minimum Eigenvalue (MME) detection method}

The steps of the MME algorithm are stated below

- Compute the received sample covariance matrix

$$
\mathbf{R}_{\mathbf{r}}\left(\mathbf{N}_{\mathbf{s}}\right)=\frac{1}{\mathbf{N}_{\mathbf{s}}} \mathbf{Y} \mathbf{Y}^{*}
$$

- Compute the Maximum and Minimum Eigenvalues of the matrix $\mathbf{R}_{\mathbf{r}}\left(\mathbf{N}_{\mathbf{s}}\right)$ (i.e. $\lambda_{\max }$ and $\lambda_{\min }$ ).

- Sensing decision is given by:

$$
\mathcal{D}=\left\{\begin{array}{lll}
\mathcal{H}_{1} & \text { if } & \frac{\lambda_{\max }}{\lambda_{\min }} \geq \gamma_{M M E} \\
\mathcal{H}_{0} & \text { otherwise } &
\end{array}\right.
$$

Note that $\gamma_{M M E}$ represents the threshold for the MME method. It is shown that the covariance matrix of the received signal in the absence of any signal at the receiver approximates to one class of random matrices called Wishart random matrix. The probability density function of Wishart random matrix has no marginal defined expression, finite dimensional case, and also present complex mathematics. Some studies on the spectral distribution of eigenvalues found the limiting values for maximum and minimum eigenvalues. Based on the proves given in [6], the distribution of the (properly rescaled) largest eigenvalue of the complex (real) Wishart matrix converges to 
the Tracy-Widom law as $M, N_{s}$ tend to $+\infty$ in some ratio $M / N_{s}>0$. As a result, the threshold $\gamma_{M M E}$ given by:

$$
\gamma_{M M E}=\frac{\chi^{2}}{\delta^{2}}\left(1+\frac{\chi^{-2 / 3}}{\left(M N_{s}^{2}\right)^{1 / 6}} F_{\beta}^{-1}\left(1-P_{f a}\right)\right)
$$

Where $\chi=\sqrt{N_{s}}+\sqrt{M N_{s}}, \delta=\sqrt{N_{s}}-\sqrt{M N_{s}}$, and $F_{\beta}$ is the cumulative distribution function (CDF) of the TracyWidom distribution of order $\beta$ (i.e. $\beta=1$ for real signal, $\beta=2$ for complex signal).

\section{B. Energy with Minimum Eigenvalue (EME) detection method}

The steps of the EME algorithm are stated below

- Compute the covariance matrix $\mathbf{R}_{r}$ as given in (4).

- Compute the average power of the received signal

$$
\Gamma\left(N_{s}\right)=\frac{1}{M N_{s}} \sum_{i=1}^{M} \sum_{n=0}^{N_{s}-1}\left|r_{i}[n]\right|^{2}
$$

- Then the eigenvalues of $\mathbf{R}_{\mathbf{r}}$ are obtained.

- Decision: if

$$
\frac{\Gamma\left(N_{s}\right)}{\lambda_{\min }}>\gamma_{E M E}
$$

where $\gamma_{E M E}$ represents the threshold for EME method defined by:

$$
\gamma_{E M E}=\left(\sqrt{\frac{2}{M N_{s}}} Q^{-1}\left(P_{f a}\right)+1\right) \frac{N_{s}}{\delta^{2}}
$$

where $Q($.$) is the Gaussian Q-function and P_{f a}$ is the probability of false alarm.

In the above discussion, we can notice that the threshold are not depending on the noise property but rather on the probability of false alarm, the number of segments $M$ and the number of samples $N_{s}$ per segment.

\section{Cyclostationary Based Detection: SPCAF ALGORITHM}

In wireless communications, the transmitted signals show very strong cyclostationary features [12]. In the context of spectrum sensing many works have been conducted in using the cyclostationary features to detect the presence of PU in the radio environment [4]. In general, this method can perform better than the eigenvalue based detectors. However its main drawbacks are the complexity associated with the detection technique and needs of some a-priori knowledge of the PU signal.

The cyclostationary detector can be realized by analysing the Cyclic Autocorrelation Function (CAF) of a received signal $r(k)$. The CAF of a received signal $r(k)$ at the SU can be expressed as illustrated in (7).

$$
R_{r}(k, \tau)=\sum_{\alpha} R_{r}^{\alpha}(\tau) \mathrm{e}^{2 \pi j \alpha k}
$$

where $\tau$ is lag associated to the autocorrelation function, $\alpha$ the cyclic frequency and $R_{r}^{\alpha}(\tau)$ is given by (8).

$$
R_{r}^{\alpha}(\tau)=\lim _{N_{s} \rightarrow \infty} \frac{1}{N_{s}} \sum_{k=0}^{N_{s}-1} R_{r}(k, \tau) \mathrm{e}^{-2 \pi j \alpha k}
$$

The discrete-time consistent and unbiased estimation of the $\mathrm{CAF}$ of a random process is given as:

$$
\tilde{R}_{r r^{*}}^{\alpha}(\tau)=\frac{1}{N_{F F T}} \sum_{k=0}^{N_{F F T}-1} r(k) r^{*}(k+\tau) \mathrm{e}^{-2 j \pi \alpha k}
$$

For a given lag parameter $\tau \in\{1,2, \ldots, L\}$, the cyclic autocorrelation function (CAF) can be seen as Fourier transform of $V=\left[r(0) r^{*}(0+\tau), r(1) r^{*}(1+\tau), \ldots, r\left(N_{F F T}-\right.\right.$ 1) $\left.r^{*}\left(N_{F F T}-1+\tau\right)\right]$, where $N_{F F T}$ is FFT size. As shown in the work of Khalaf et al. [8], the CAF is an $N_{F F T}$-dimensional sparse vector in cyclic frequency domain for a fixed lag parameter $\tau$. Moreover, it presents a symmetry property as illustrated in (10).

$$
\left\|\tilde{R}_{r r^{*}}^{\alpha}(\tau)\right\|_{2}=\left\|\tilde{R}_{r r^{*}}^{-\alpha}(\tau)\right\|_{2}
$$

Using a compressed sensing (CS) recovery technique like the Orthogonal Matching Pursuit (OMP) algorithm [13], we can accurately estimate the CAF using a limited and small number of received samples $N_{s}<<N_{F F T}$. If the obtained CAF verifies the property (10) then $\mathcal{H}_{1}$ is true otherwise $\mathcal{H}_{0}$ is true. Its important to note that even under $\mathcal{H}_{0}$ the obtained CAF verifies the symmetry property. However, when using a small number of samples, the probability to obtain a symmetrical CAF under $\mathcal{H}_{0}$ is very small [8]. This SPCAF technique, can perform with a limited number of samples and consequently with lower complexity and shorter observation time compared to the classical cyclostationary feature detector.

\section{SPECTRUM SENSING EXPERIMENTAL SETUP}

The performance of the previously presented spectrum sensing algorithms has been verified by conducting experiments realized by means of Universal Software Radio Peripheral (USRP) N210 board by Ettus Research. Being the realization of the SDR concept, USRP are steered from the software level, i.e. the whole data processing in the base-band is realized on the computer side. In our experiments two USRP boards have been utilized: the PU signal has been generated by means of the first board, whereas the second one has been used for spectrum sensing purposes and acted as the secondary user. The whole software processing has been realized in the opensource GNU-Radio environment. This set of libraries together with the appropriate drivers for manipulating the USRP N210 boards and graphical programming environment allowed for implementing the selected spectrum sensing algorithms. The schematic diagram of the experimentation setup is presented in Fig. 1 and the testbed environment is shown in Fig. 2.

\section{A. Transmitter side (PU)}

At the transmitter side, three types of signals were generated, the narrow-band FM, 8QPSK and GMSK signals. As it has already been mentioned, the whole baseband processing has been realized on the computer in the GNU-Radio environment where the whole system is built from blocks. After proper power adjustment, the signal was sent to the USRP block (USRP Sink), responsible for sending data to the USRP 


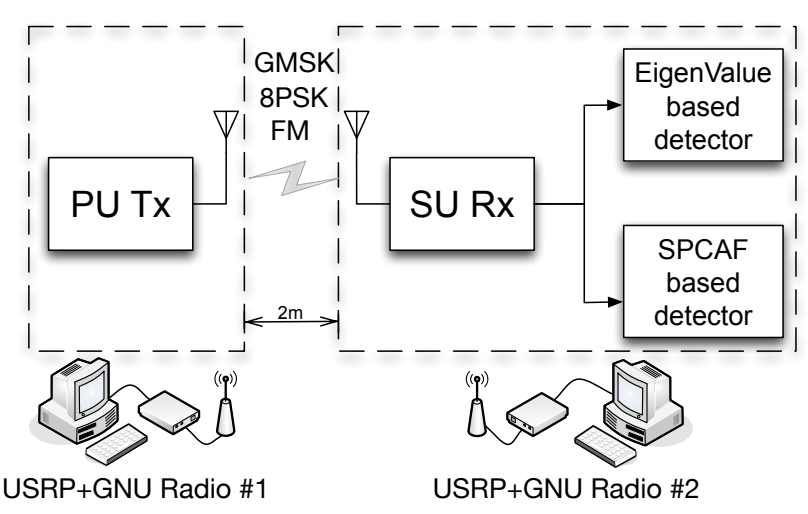

Fig. 1. Schematic system diagram

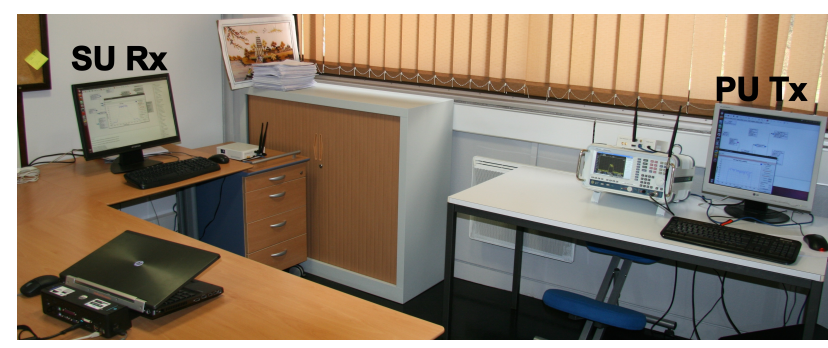

Fig. 2. The experimentation setup

platform. The center frequency was set to $560 \mathrm{MHz}$. This frequency band has been chosen intentionally - it is within the TV band and is not occupied in the physical location where the experiment was conducted (i.e. no interference from the distance digital-television station could be observed). The spectrum bandwidth occupied by the FM, 8PSK and GMSK signals are $144 \mathrm{kHz}, 600 \mathrm{kHz}$ and $800 \mathrm{kHz}$, respectively.

\section{B. Receiver side (SU)}

As indicated in Fig. 1, the three spectrum sensing algorithms explained in Section II have been implemented. Analogously to the transmitter side, the whole baseband processing that will be performed by the SU - has been realized in the computer side using the GNU-Radio environment. The schematic diagram of the receiver is shown in Fig. 3. One can observe the presence of the USRP Source block responsible for delivering data from RF spectrum to the computer; it operates at the center frequency equal to $560 \mathrm{MHz}$ and covers the band of $1 \mathrm{MHz}$ (what corresponds to complex sampling frequency equal to $1 \mathrm{Msps}$ ). In order to evaluate the influence of noise on the performance of selected spectrum sensing algorithms, additional block for noise generation has been used and the noise-signal of appropriate power has been added to the signal produced by the USRP Source block. After, the signal is split into three parallel chains: one dedicated for each sensing method algorithm. In such a configuration the three algorithms operate on the same received samples making the comparison fair. One can also observe the presence of the FFT plot block used for displaying the received signal on the computer screen. In the upper processing chain, devoted for cyclostationary feature spectrum sensing algorithm, the signal is converted from complex to real type and such modified signals are subject to processing in the Symmetry block, realizing the functionality of the SPCAF algorithm described in the previous sections. The lower processing chains, devoted for eigenvalue based algorithm realized by the EBSS_algo block. In this block, the parameter Sensing Algo selects the detection method: " 0 " for MME and " 1 " for EME.

\section{EXPERIMENTAL RESULTS}

In order to compare the performance of the selected spectrum sensing algorithms let us analyse the results obtained during the conducted experiments.

\section{A. FM signal as $P U$}

Here, we compare performance of the SPCAF based blind detector with the MME and EME detectors. A frequency modulated signal is used as primary user's signal. In the experiments, the central carrier frequency is set to $560 \mathrm{MHz}$. We compute the correct detection probability $\left(P_{d}\right)$ for the three detectors at different values of estimated SNR. In order to estimate the SNR, the noise power $\sigma^{2}$ is estimated at the receiver with no transmitted signal. Then, the transmitter is switched on and its transmission power is varied to obtain different signal-to-noise ratios (SNRs) at the receiver. Fig. 4 shows the detection probability of the three detectors obtained through experiments as function of SNRs. As concluded from the measurements, the probability of false alarm $\left(P_{f a}\right)$ is approximately equal to 0.08 for both detectors. Furthermore, for the SPCAF detector, the maximum value of the lag parameter is $\tau=5$ and the FFT size is $N_{F F T}=2048$. It is clear from Fig. 4 that the performance of the SPCAF is significantly better than the MME and EME algorithm. MME and EME methods show similar performance. Another important point to note is that the number of received samples used by SPCAF is $N_{s}=512$.

\section{B. 8PSK signal as $P U$}

In this section we test the performance of the three algorithm for a 8PSK signal. The central carrier frequency is set to 560 $\mathrm{MHz}$. We compute the correct detection probability $\left(P_{d}\right)$ for the three detectors at different values of estimated SNR. The probability of false alarm $\left(P_{f a}\right)$ is approximately equal to 0.08 for both detectors. Furthermore, for the SPCAF detector, the maximum value of the lag parameter is $\tau=5$ and the FFT size is $N_{F F T}=2048$. Fig. 5 shows that the performance of the SPCAF is significantly better than the MME and EME algorithm. An important point to note here is that the performance of MME method is better than the EME method. The number of received samples used by SPCAF is $N_{s}=512$.

\section{GMSK signal as $P U$}

In this part, the primary user signal is a GMSK (Gaussian Minimum Shift Keying) signal. Fig. 6 shows the detection 


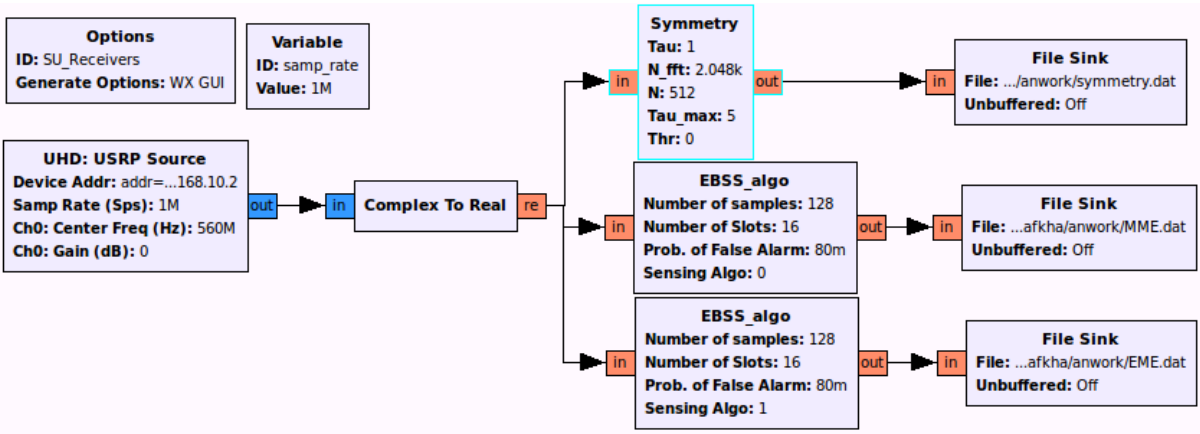

Fig. 3. Diagram of the SU receiver realized in the GNU radio (Screenshot from GRC)

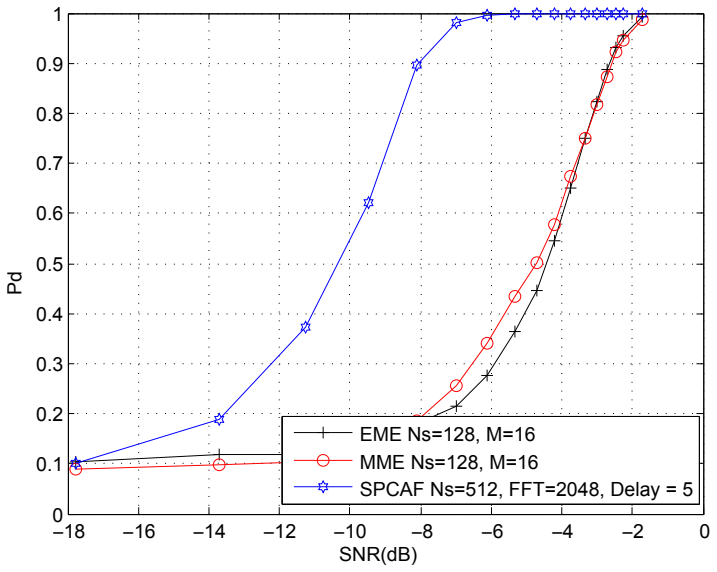

Fig. 4. Probability of detection for the three algorithms when using FM signal as PU $\left(P_{f a}=0.08\right)$.

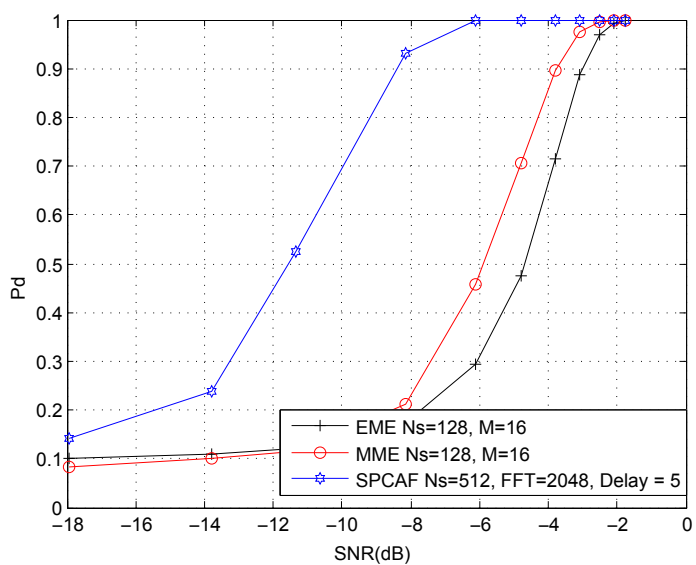

Fig. 5. Probability of detection for the three algorithms when using 8PSK signal as PU $\left(P_{f a}=0.08\right)$.

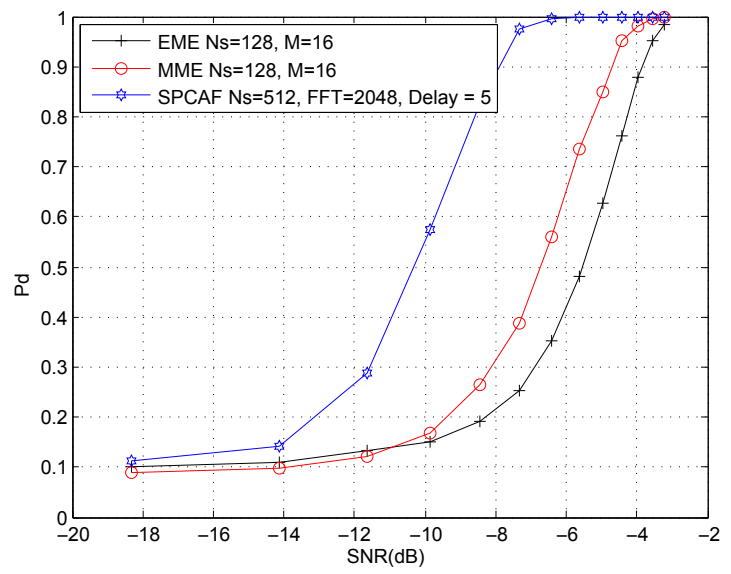

Fig. 6. Probability of detection for both algorithms when using GMSK signal as PU $\left(P_{f a}=0.08\right)$

probability achieved by the secondary user using SPCAF, MME and EME, while maintaining the false alarm probability below 0.08. Based on the results in Fig. 6, it can be concluded that the SPCAF outperforms the eigenvalue based MME and EME methods at low signal-to-noise ratios (SNRs).

\section{CONCLUSION}

In this paper, two classes of sensing methods are presented i.e. eigenvalue based detection methods (MME and EME) and SPCAF method which is based on cyclostationary feature detection. We analysed the performance of these spectrum sensing methods by measuring the detection probabilities as a function of SNR for a given false alarm probability. The testbed setup for the comparison of the three methods is based on two USRP N210 boards and GNU-Radio development toolkit using three types of modulations i.e. FM, 8PSK and GMSK. The cyclostationary feature-based detector achieves results far superior than the eigenvalue based methods in the case of finite received samples. The SPCAF gain in terms of the SNR with respect to eigenvalues-based detectors to achieve $P_{f a}=0.08$ is at least $4 \mathrm{~dB}$. This is due to the fact that 
eigenvalue based techniques are more sensitive to the number of received samples.

\section{ACKNOWLEDGEMENTS}

This work has been supported by the European Commission in the framework of the FP7 Network of Excellence in Wireless COMmunications NEWCOM\# (FP7 Contract Number: 318306).

\section{REFERENCES}

[1] I. F. Akyildiz, X. Wang, and W. Wang, "Wireless mesh networks: a survey," Computer Networks, vol. 47, no. 4, pp. 445 - 487, 2005.

[2] S. Haykin, D. J. Thomson, and J. H. Reed, "Spectrum sensing for cognitive radio," Proceedings of the IEEE, vol. 97, no. 5, pp. 849-877, 2009.

[3] Y. Zeng, Y.-C. Liang, A. T. Hoang, and R. Zhang, "A review on spectrum sensing for cognitive radio: challenges and solutions," EURASIP Journal on Advances in Signal Processing, vol. 2010, p. 2, 2010.

[4] E. Axell, G. Leus, E. G. Larsson, and H. V. Poor, "Spectrum sensing for cognitive radio: State-of-the-art and recent advances," Signal Processing Magazine, IEEE, vol. 29, no. 3, pp. 101-116, 2012.

[5] L. Lu, X. Zhou, U. Onunkwo, and G. Y. Li, "Ten years of research in spectrum sensing and sharing in cognitive radio," EURASIP Journal on Wireless Communications and Networking, vol. 2012, no. 1, pp. 1-16, 2012.

[6] Y. Zeng and Y.-C. Liang, "Maximum-minimum eigenvalue detection for cognitive radio," in Personal, Indoor and Mobile Radio Communications, 2007. PIMRC 2007. IEEE 18th International Symposium on, Sept 2007, pp. 1-5.

[7] P. Cheraghi, Y. Ma, and R. Tafazolli, "A novel blind spectrum sensing approach for cognitive radios," in PGNET 2010 Conference, 2010

[8] Z. Khalaf, A. Nafkha, and J. Palicot, "Blind spectrum detector for cognitive radio using compressed sensing and symmetry property of the second order cyclic autocorrelation," in Cognitive Radio Oriented Wireless Networks and Communications (CROWNCOM), 2012 7th International ICST Conference on. IEEE, 2012, pp. 291-296.

[9] H. Urkowitz, "Energy detection of unknown deterministic signals," Proceedings of the IEEE, vol. 55, no. 4, pp. 523-531, April 1967.

[10] Y. Zeng and Y.-C. Liang, "Eigenvalue-based spectrum sensing algorithms for cognitive radio," Communications, IEEE Transactions on vol. 57, no. 6, pp. 1784-1793, June 2009.

[11] L. Cardoso, M. Debbah, P. Bianchi, and J. Najim, "Cooperative spectrum sensing using random matrix theory," in Wireless Pervasive Computing, 2008. ISWPC 2008. 3rd International Symposium on, May 2008, pp 334-338.

[12] W. A. Gardner, "Exploitation of spectral redundancy in cyclostationary signals," Signal Processing Magazine, IEEE, vol. 8, no. 2, pp. 14-36, 1991.

[13] T. T. Cai and L. Wang, "Orthogonal matching pursuit for sparse signal recovery with noise," Information Theory, IEEE Transactions on, vol. 57, no. 7, pp. 4680-4688, 2011. 